\title{
Effect of Rayleigh-scattering distributed feedback on multiwavelength Raman fiber laser generation
}

\author{
A. E. El-Taher, ${ }^{1, *}$ P. Harper, ${ }^{1}$ S. A. Babin, ${ }^{2}$ D. V. Churkin, ${ }^{2}$ E. V. Podivilov, ${ }^{2}$ \\ J. D. Ania-Castanon, ${ }^{3}$ and S. K. Turitsyn ${ }^{1}$ \\ ${ }^{1}$ Photonics Research Group, Aston University, Birmingham, B4 7ET, UK \\ ${ }^{2}$ Institute of Automation and Electrometry, SB RAS, Novosibirsk 630090, Russia \\ ${ }^{3}$ Instituto de Óptica, Consejo Superior de Investigaciones Cientificas, C/ Serrano 121, Madrid 28006, Spain \\ *Corresponding author: eltaheae@aston.ac.uk
}

Received July 27, 2010; revised November 23, 2010; accepted November 25, 2010; posted December 2, 2010 (Doc. ID 132300); published January 6, 2011

We experimentally demonstrate a Raman fiber laser based on multiple point-action fiber Bragg grating reflectors and distributed feedback via Rayleigh scattering in an $\sim 22-\mathrm{km}$-long optical fiber. Twenty-two lasing lines with spacing of $\sim 100 \mathrm{GHz}$ (close to International Telecommunication Union grid) in the C band are generated at the watt level. In contrast to the normal cavity with competition between laser lines, the random distributed feedback cavity exhibits highly stable multiwavelength generation with a power-equalized uniform distribution, which is almost independent on power. (c) 2011 Optical Society of America

OCIS codes: $190.5650,140.3550,290.5870$.

Multiwavelength fiber lasers have attracted great interest owing to their wide applications in wavelength-divisionmultiplexing (WDM) systems, instrument testing, optical fiber sensors, and spectroscopy. Several techniques have been proposed to ensure stable multiwavelength laser operation based on semiconductor optical amplifiers [1] , erbium-doped fiber lasers [2], spectrum sliced supercontinuum [3], or Raman fiber lasers (RFLs) [4-10]. The RFL-based multiwavelength sources are very attractive because of their broad and flat gain at many customizable wavelengths. Different types of multiwavelength RFLs, mainly in ring cavity configurations, have been proposed and demonstrated utilizing multichannel filters based on Fabry-Perot [4-6] or Sagnac [7] interferometers. As a result, multiwavelength RFLs can emit up to 58 stable lines simultaneously [4] in different spectral bands [7]. Fiber Bragg gratings (FBGs) reflecting several wavelengths being either multimode [8], phase-shifted [9], or sampled [10] have also been demonstrated for multiwavelength operation in RFLs with linear cavities but generally with a relatively low number of spectral lines.

The basic principles of operation of such multiwavelength RFLs are, however, still rather uncertain. Simple explanations, such as inhomogeneous saturation of the Raman gain spectrum, contradict the direct measurements of the saturated gain [11]. The mechanism of inhomogeneity is shown to be defined by nonlinear effects, namely, by four-wave mixing (FWM) between generated spectral components either interchannel (between generated lines) or intrachannel (between longitudinal cavity modes within one line). Since the integral (over frequency) effect of FWM is zero, it leads to a redistribution of power from the central to weak side components. This FWM-induced spectral inhomogeneity was initially attributed to inhomogeneous losses $[\underline{5}, 6]$ and then to inhomogeneous gain [12] that is shown to be more correct in the frame of the developed statistical model. Though the classification of inhomogeneity is a rather academic problem, the main common conclusion $[5,6,12]$ is that the integral FWM-induced losses depend on the line intensity and spectral width of the line-selecting filter/reflector.
Such nonlinear losses result both in spectral broadening of the individual line and in power equalization between the lines at multiwavelength operation.

Note that all multiwavelength RFL schemes [4-10] are based on long fibers (4-50 km), so Rayleigh scattering (RS) may play an important role. Stable narrow-linewidth lasing utilizing RS-based random distributed feedback (RDFB) only (without regular reflectors) has been demonstrated recently [13]. A distributed RS mirror has also been used to improve the generation of Brillouin combs in a linear RFL cavity, see, e.g., [14,15]. Similarly, it may be utilized as a broadband mirror in multiwavelength RFLs, and cw generation of five lines has been obtained in a cavity formed by an RS mirror and five FBGs [16]. Stable operation with $\sim 1 \mathrm{~nm}$ separation between the lines appears possible in schemes involving a photonic crystal fiber loop mirror [17] or two different-wavelength FBGs placed at opposite ends of a $200-\mathrm{km}$-long fiber [18]. In this Letter, we explore the opportunity of achieving stable uniform generation of multiple lines with $0.8 \mathrm{~nm}$ spacing (close to International Telecommunication Union $100 \mathrm{GHz}$ grid) in a cavity formed by an RS-based distributed mirror and an array of FBGs in a simple linear scheme. To clarify the specifics of the RDFB, we perform a direct comparison with a laser based on broadband $4 \%$ reflection from the cleaved fiber end.

A single pump laser at $1455 \mathrm{~nm}$, see Fig. 1, is coupled into the fiber span through a $1450 / 1550 \mathrm{~nm}$ WDM coupler to induce broad Raman gain in the $1550 \mathrm{~nm}$ C-band region. The laser cavity is formed by RDFB, owing to the Raman amplified Rayleigh backscattering along the fiber,

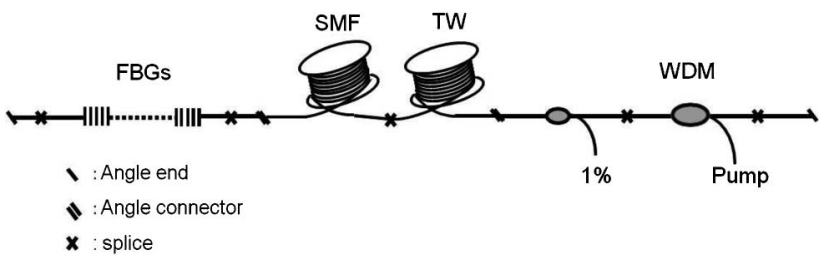

Fig. 1. Schematic depiction of the experimental setup. 
and an array of 22 highly reflective FBGs placed at the left fiber end with a $0.3 \mathrm{~nm}$ reflection bandwidth a maximum reflection of $\sim 95 \%$ each, spaced by $\sim 0.8 \mathrm{~nm}$ $(\sim 100 \mathrm{GHz})$ to cover a wavelength range of 1552 $1570 \mathrm{~nm}$. Parasitic feedback from the fiber ends and connections is avoided by using angled fiber end facets and angled polished connectors. A splitter with a $1 \%$ monitor port was inserted near the right fiber end to measure the Raman laser output power and spectra measured with a high-resolution $(\sim 0.01 \mathrm{~nm})$ optical spectrum analyzer. For a power-equalized multiwavelength operation, one should first flatten the Raman gain spectral profile. Usually, multiwavelength pumping is used to reduce gain variance over the spectrum; we have used another approach: the cavity is formed by fibers of different types $-11 \mathrm{~km}$ of SMF-28 and $10.5 \mathrm{~km}$ of TrueWave (TW) fibers with Raman gain coefficients 0.38 and $0.593(* 1 / \mathrm{W} \mathrm{km})$, correspondingly [19]. In highly nonlinear TW fiber having a zero-dispersion wavelength near $1456 \mathrm{~nm}$, the $1455 \mathrm{~nm}$ pump wave is significantly broadened [20]; see Fig. 2 . The broadband pump results in a smoothed Raman gain spectrum with variation of less than $3 \mathrm{~dB}$ in the $1550-1570 \mathrm{~nm}$ wavelength range at pump powers $0.9-2.75 \mathrm{~W}$; see inset in Fig. 2. The spectrum in pure SMF having $\sim 10 \mathrm{~dB}$ nonuniformity is also shown for comparison.

The setup exhibits clear laser behavior with a threshold pump power of $\sim 0.8 \mathrm{~W}$, Fig. 3 . The total output power from the right fiber end is as high as $1.6 \mathrm{~W}$, demonstrating more than $40 \%$ generation efficiency. The power variations are small both for the total power and for the individual laser lines. The laser simultaneously delivers stable laser radiation at all the $22 \mathrm{FBG}$ wavelengths starting from the threshold [Fig. 4(a)] up to the maximum power [Fig. 4(b)] with background ASE suppression of $\sim 25 \mathrm{~dB}$. The individual linewidths (FWHM) are less than $0.3 \mathrm{~nm}$, so the frequency comb is contrasted. The distribution follows the Raman gain curve with two maxima at $\sim 1555$ and $\sim 1565 \mathrm{~nm}$ and a small dip between them. With increasing power, only limited change in the spectral shape was observed.

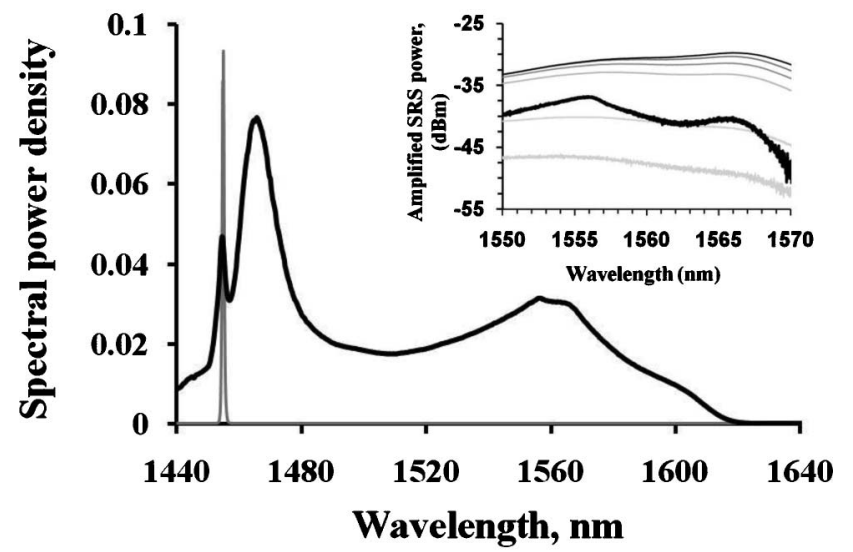

Fig. 2. Comparison between pump power spectrum in linear scale before (gray curve) and after (black curve) TW fiber at $1.8 \mathrm{~W}$ pump power. Inset, backward measured amplified SRS (FBGs are not installed) as the pump power is increased from 0.9 to $2.75 \mathrm{~W}$ for TW and SMF fibers spliced together, and the thick black curve is the same for the SMF fiber of equivalent length, $22 \mathrm{~km}$, at $1.8 \mathrm{~W}$.
To justify the role of the RS-based RDFB, we arrange a conventional linear cavity between highly reflective FBGs and 4\% Fresnel reflection inserted by means of flat cleaving of the fiber end. As expected, the generation threshold for this cavity is lower [see Fig. 3 (circles)] because of the much higher reflection providing highercavity $Q$ factor. However, the slope efficiency for the flat end is lower, and thus the highest achievable output power is slightly lower than that for the pure RDFB cavity.

The generation spectra measured with a flat end cavity reveal nonuniform distributions strongly changing with power, see Fig. 4 (gray curves). At low pump power, the output spectrum consists of two different groups of lines generated near the Raman gain spectral maxima, see Fig. 4(a). The generation at wavelengths near the local minimum is suppressed, contrary to the case of RDFB cavity. With increasing pumping the power maximum shifts to longer wavelengths owing to the effect of cascaded Raman scattering discussed earlier [5]. The distribution becomes flatter but less uniform: the difference between line intensities is $>30 \mathrm{~dB}$, which is much greater than that for RDFB cavity, whereas the comb contrast is similar $(\sim 10 \mathrm{~dB})$. At the same time, the amplified spontaneous emission suppression is greater than $40 \mathrm{~dB}$.

The mechanisms responsible for the observed RDFB effects in multiwavelength Raman lasers can be considered as follows. First, the observed improved power equalization between the generated lines in the RDFB cavity is certainly defined by unique features of RS-based RDFB: spectral selectivity with near-Gaussian statistics [21] in the absence of longitudinal modes [13]. In a conventional cavity, the modes formed by point reflectors have equidistant frequencies and a rather uniform power distribution along the cavity, thus leading to competition between the modes (or lines) utilizing the same pump, whereas in the pure RDFB laser the generated radiation is random in frequency and space. One can define only the average longitudinal distribution of such "random modes" that is mainly defined by the Raman gain [13]. We suppose that at multiwavelength operation of the RDFB laser, the different lines may have a sufficiently

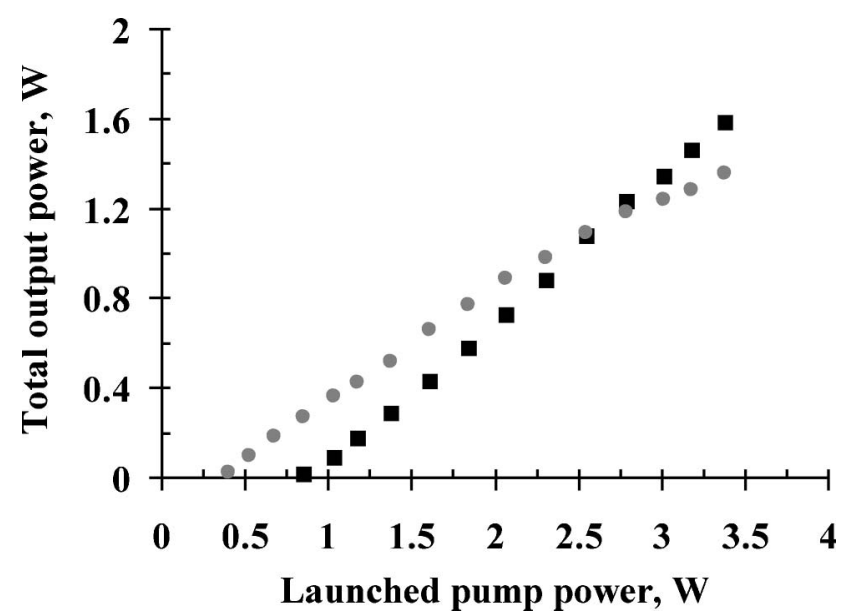

Fig. 3. Laser output power as a function of pump power: squares are the output power values for the angle end, and circles are the output power values with the flat end. 

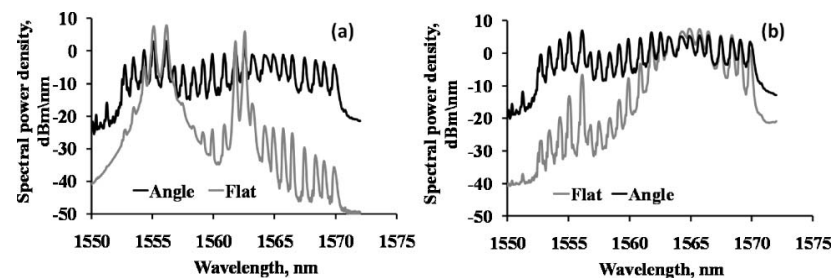

Fig. 4. Optical spectra at various output power for both angle (black) and flat (gray) end setups: (a) at $0.28 \mathrm{~W}$, (b) at $1.35 \mathrm{~W}$.

different distribution along the fiber to reduce their competition self-consistently. Note that a similar effect was observed for a Brillouin comb which becomes uniform with the assistance of RS at high pump power [15].

The second important fact is that the total power of the pure RDFB cavity becomes equal and even slightly higher than that for a normal cavity, in spite of higher linear losses, which can be attributed to nonlinear losses. As the fiber length of $\sim 22 \mathrm{~km}$ is close to the estimated length of distributed RS mirror [13], the RDFB effect on generation becomes comparable with the Fresnel reflection at high Raman gain. At nearly equal output power, the variation of power along the fiber is quite different in two cases: in case of pure RDFB the power at the left end is much lower than that for the $4 \%$ reflection, meaning lower nonlinear losses on the FBGs. We have directly measured the output power and spectra at the left end confirming much higher intensity and corresponding nonlinear losses at the FBGs in case of the Fresnel cavity. The lost power is close to the observed difference in the output power. At the same time, the cascaded Raman shift is defined by the intensity integral along the fiber that is larger for the $4 \%$ cavity. For quantitative analysis of the effects, numerical simulations of the spatial distributions in both cases are needed.

In conclusion, we have shown that cw multiwavelength generation with $40 \%$ efficiency is easily achieved in telecommunication fiber owing to the random distributed Rayleigh-scattering feedback. The power in all lines is equalized thanks to a gain spectrum flattened by means of pump spectral broadening. The remaining nonuniformity can be eliminated by optimizing the FBG reflectivity, which is not possible in schemes with loop mirrors and interferometers. The number of generated lines is limited in our case by the number of available FBGs; however, we anticipate that this can be easily increased beyond 50 using additional FBGs. This technique can also be applied for other spectral bands using corresponding pump and FBGs wavelengths. The proposed multiwavelength Raman fiber laser has potential uses in telecommunications and sensing.
The authors acknowledge financial support of the Engineering and Physical Sciences Research Council (EPSRC) EP/E015646/1 and the Russian Ministry of Education and Science grants and thank I. N. Nemov for fabrication of the FBG array.

\section{References}

1. N. Pleros, C. Bintjas, M. Kalyvas, G. Theophilopoulos, K. Yiannopoulos, S. Sygletos, and H. Avramopoulos, IEEE Photon. Technol. Lett. 14, 693 (2002).

2. A. Bellemare, M. Karasek, M. Rochette, S. L. Rochelle, and M. Tetu, J. Lightwave Technol. 18, 825 (2000).

3. F. Futami and K. Kikuchi, IEEE Photon. Technol. Lett. 13, 73 (2001).

4. N. S. Kim and X. Zou, in Proceedings of the Optical Fiber Communication Conference and Exhibit (Optical Society of America, 2002), pp. 640-641.

5. Q. Wang, Y. Wang, W. Zhang, X. Feng, X. Liu, and B. Zhou, Opt. Lett. 30, 952 (2005).

6. Q. Wang, X. Liu, L. Xing, X. Feng, and B. Zhou, Opt. Lett. 30, 3033 (2005).

7. C.-S. Kim, R. M. Sova, and J. U. Kang, Opt. Commun. 218, 291 (2003).

8. Y. Han, S. B. Lee, D. S. Moon, and Y. Chung, Opt. Lett. 30, 2200 (2005).

9. Y.-G. Han, T. V. A. Tran, S.-H. Kim, and S. B. Lee, Opt. Lett. 30, 1114 (2005).

10. Z. Wang, Y. Cui, B. Yun, and C. Lu, IEEE Photon. Technol. Lett. 17, 2044 (2005).

11. S. A. Babin, D. V. Churkin, S. I. Kablukov, and E. V. Podivilov, Opt. Express 13, 6079 (2005).

12. S. A. Babin, D. V. Churkin, A. E. Ismagulov, S. I. Kablukov, and E. V. Podivilov, J. Opt. Soc. Am. B 24, 1729 (2007).

13. S. K. Turitsyn, S. A. Babin, A. E. El-Taher, P. Harper, D. V. Churkin, S. I. Kablukov, J. D. Ania-Castañón, V. Karalekas, and E. V. Podivilov, Nat. Photon. 4, 231 (2010).

14. A. K. Zamzuri, M. I. Md. Ali, A. Ahmad, and R. Mohamad, Opt. Lett. 31, 918 (2006).

15. A. K. Zamzuri, M. H. Al-Mansoori, N. M. Samsuri, and M. A. Mahdi, Appl. Opt. 49, 3506 (2010).

16. O. Frazão, C. Correia, J. L. Santos, and J. M. Baptista, Meas. Sci. Technol. 20, 045203 (2009).

17. A. M. R. Pinto, O. Frazão, J. L. Santos, and M. Lopez-Amo, Appl. Phys. B 99, 391 (2010).

18. A. E. El-Taher, M. Alcon-Camas, S. A. Babin, P. Harper, J. D. Ania-Castañón, and S. K. Turitsyn, Opt. Lett. 35, 1100 (2010).

19. C. Fludger, A. Maroney, N. Jolley, and R. Mears, in Proceedings of the Optical Fiber Communication Conference (Optical Society of America, 2000), Vol. 37, pp. 100-102.

20. T. J. Ellingham, J. D. Ania-Castañón, and S. K. Turitsyn, Opt. Commun. 257, 176 (2006).

21. A. A. Fotiadi and R. V. Kiyan, Opt. Lett. 23, 1805 (1998). 